\title{
Conhecimento dos fatores de risco modificáveis para doença cardiovascular entre mulheres e seus fatores associados: um estudo de base populacional
}

\author{
Knowledge of modifiable risk factors for cardiovascular disease \\ among women and the associated factors: a population-based study
}

Gabriel Missaggia Bonotto ${ }^{1}$ Raul Andres Mendoza-Sassi ${ }^{2}$ Lulie Rosane Odeh Susin ${ }^{2}$

\footnotetext{
${ }^{1}$ Pró-Reitoria de Assuntos Estudantis e Comunitários, Universidade Federal do Pampa. R. Vereador Alberto Benevenuto 3200, Bairro do Passo. 97670-000 São Borja RS Brasil.gabrielmissaggia@ yahoo.com.br

${ }^{2}$ Faculdade de Medicina, Universidade Federal do Rio Grande.
}

\begin{abstract}
The scope of this study was to evaluate the knowledge of modifiable risk factors for cardiovascular disease and its distribution in terms of demographic, socioeconomic, behavioral and biological factors among women living in the State of Rio Grande. It was a cross-sectional population-based study, with the inclusion of women aged 18 years and over. Descriptive, bivariate and multivariable Poisson Regression analysis were performed. The outcome was defined as knowing three or more of the seven risk factors studied (75th percentile). Of the 1,593 respondents, 33\% knew three or more factors. The outcome was independently associated with increased likelihood of knowledge among subjects in the 25-44 years old age group, non-white skin color, complete secondary education, higher income and having cardiovascular disease. On the other hand, sedentary women were less likely to have such knowledge. These findings indicate the need for educational activities for enhancing comprehension and knowledge of the factors associated with cardiovascular disease among women, particularly among the poorest and less educated.
\end{abstract}

Key words Knowledge, Health attitudes and practices, Risk factors, Cardiovascular diseases, Women
Resumo O objetivo deste estudo foi avaliar o conhecimento dos fatores de risco modificáveis para a doença cardiovascular e sua distribuição em termos demográficos, socioeconômicos, comportamentais e biológicos entre mulheres residentes em Rio Grande, Rio Grande do Sul, Brasil. Foi um estudo transversal de base populacional, com a inclusão de mulheres com idade igual ou superior a 18 anos. Foram realizadas análise descritiva, bivariada e multivariável pela Regressão de Poisson. O desfecho foi definido como conhecer três ou mais dos sete fatores de risco pesquisados (percentil 75). De 1.593 entrevistadas, 33,0\% conheciam três ou mais fatores. Foram associados de forma independente com maior probabilidade de conhecimento: a faixa etária de 25 a 44 anos, cor da pele não branca, ensino médio completo, maior renda e ser portadora da doença cardiovascular. Mulheres sedentárias apresentaram menor probabilidade de conhecimento. Os achados indicam a necessidade de ações educativas para melhorar a compreensão e o conhecimento dos fatores associados à doença cardiovascular, particularmente entre as mulheres mais pobres e menos escolarizadas.

Palavras-chave Conhecimento, Conhecimentos, atitudes e práticas em saúde, Fatores de risco, Doenças cardiovasculares, Mulheres 


\section{Introdução}

As doenças crônicas não transmissíveis (DCNT) são atualmente um grave problema de saúde pública, sendo responsáveis por 72,0\% das mortes no Brasil. Entre elas, destaca-se a doença cardiovascular (DCV) como a principal causa de morte e incapacidade no Brasil e em muitos países, acarretando mais de sete milhões de óbitos por ano em todo o mundo, principalmente em grupos vulneráveis, como idosos, mulheres, pessoas de menor renda e escolaridade ${ }^{1-3}$.

A tendência crescente da incidência e prevalência observada para esta doença, nos últimos anos, obedece principalmente às mudanças no estilo de vida e ao envelhecimento da população ${ }^{4}$. É esperado, segundo estimativas para 2020, que a DCV seja responsável por 25 milhões de mortes no mundo ${ }^{1}$.

No controle dos fatores de risco para DCV, visando à redução do seu impacto na saúde da população, é fundamental a identificação de pessoas portadoras desses fatores, de forma que as mesmas se mobilizem para uma mudança de comportamento. Como muitos desses fatores estão relacionados ao estilo de vida e são, portanto, modificáveis, diversas ações educativas no âmbito da saúde pública têm sido aplicadas. É o caso das políticas e programas instituídos para o incentivo à prática da atividade física, da dieta saudável, da cessação do tabagismo, além do controle das principais doenças que atuam como fatores de risco, como hipertensão arterial sistêmica (HAS) e o diabetes mellitus (DM) $)^{5-7}$.

No entanto, existem evidências de que para que essa mudança comportamental tenha o efeito esperado, as pessoas devem passar por uma etapa na qual identifiquem as consequências positivas e/ou negativas que esse comportamento e/ou exposição a fatores de risco podem acarre$\operatorname{tar}^{8,9}$. Para que isso suceda é necessário que os fatores de risco que levam a essas doenças crônicas sejam conhecidos pela população.

Estudos realizados em outros países têm mostrado que o conhecimento sobre os fatores de risco para DCV é escasso, e que as questões socioeconômicas (escolaridade e renda em particular) estão associadas ao baixo entendimento dos mesmos ${ }^{10-13}$. No Brasil, os estudos realizados, a maioria de base populacional, confirmam os achados dos outros países. Esses estudos abordam o conhecimento sobre fatores de risco para doenças crônicas isoladamente para um fator, como, por exemplo, o papel da atividade física na prevenção das DCNT ${ }^{14,15}$ ou, no máximo três.
Existem poucos estudos que analisam o conjunto de todos os fatores modificáveis envolvidos. Justifica-se essa abordagem mais ampla porque é frequente a coexistência de mais de um fator de risco para doença cardiovascular entre as pessoas ${ }^{16}$. É importante então saber qual o conhecimento que a população tem a respeito de todos os fatores de risco modificáveis que levam à DCV.

Há diferenças de gênero na prevalência de fatores de risco para DCV, como HAS e DM, sendo esta mais alta entre as mulheres ${ }^{17}$. Essa desigualdade se observa também em outros fatores de risco para DCV, como obesidade e fumo, que têm mostrado uma tendência de estabilização ou ainda de aumento entre as mulheres, ao contrário do observado nos homens, reforçando esse padrão de desigualdade entre gêneros. Além dessas diferenças, também são observadas disparidades na prevalência dos fatores de risco entre grupos socioeconômicos, mostrando que a iniquidade para os fatores de risco se estende também à classe socioeconômica ${ }^{18,19}$.

Considerando a necessidade de que a população seja consciente dos fatores que levam à DCV para poder iniciar uma mudança comportamental, a distribuição desigual dos fatores risco na população segundo gênero e grupo socioeconômico, e a importância de avaliar qual o conhecimento para todos os fatores de risco relacionados com a patogênese da DCV, foi formulado o presente estudo. Os objetivos propostos são estudar qual o conhecimento que as mulheres têm dos fatores de risco modificáveis para a DCV, e como se distribui em termos de fatores demográficos, socioeconômicos, comportamentais e de morbidades em um município do extremo Sul do Brasil. Espera-se que a informação obtida através desta pesquisa possa servir para aprimorar as ações dirigidas ao controle de DCNT no país, e em particular àquelas dirigidas às mulheres.

\section{Métodos}

Trata-se de um estudo transversal de base populacional realizado na zona urbana do município do Rio Grande, no estado do Rio Grande do Sul, Brasil. A cidade situa-se no extremo sul do estado e possuía, segundo o censo demográfico de 2010, uma população estimada de 197.228 habitantes, sendo 96\% residentes na área urbana. O município tem o $4^{\circ}$ Produto Interno Bruto (PIB) do estado e o Índice de Desenvolvimento Humano (IDH) é de $0,792^{20}$. Tem o segundo maior porto marítimo de contêineres do Brasil, um polo naval 
de plataformas de petróleo e um parque industrial de derivados da soja e de fertilizantes, entre outros.

O presente estudo é parte de um projeto de pesquisa maior realizado de abril a novembro de 2011 e intitulado Educação, conhecimento sobre fatores de risco e utilização de serviços de saúde em mulheres residentes em uma cidade do Sul do Brasil: um estudo de base populacional. Este estudo incluiu mulheres com idade igual ou superior a 18 anos residentes na zona urbana do município do Rio Grande, excluídas aquelas incapacitadas de responder ao questionário por motivos de doença mental.

O cálculo do tamanho da amostra foi feito no programa Epi Info 6.04 (Centers for Disease Controland Prevention, Atlanta, Estados Unidos), sendo considerados diversos fatores envolvidos (sociodemográficos, hábitos e costumes, morbidade e de saúde). Utilizou-se um risco relativo de 2,0, um nível de confiança de 95\%, um poder de $80 \%$, uma relação de não expostos para expostos de até 8:1 e uma prevalência esperada do desfecho nos não expostos de no mínimo $10 \%$. Com esses parâmetros, foi constatado que seria necessário uma amostra de 999 participantes. A este número foram acrescidos subsequentemente $10 \%$ para possíveis perdas, $20 \%$ para ajuste de fatores de confusão e $20 \%$ para possível efeito de delineamento, totalizando 1.582 mulheres. A partir desse número e sabendo que existiam 1,13 mulheres por domicilio, estimou-se que seria necessário visitar 1.400 residências para atingir a amostra desejada.

A amostragem foi por múltiplos estágios. Primeiro, foi realizada uma amostragem por conglomerados, sendo sorteados cinquenta setores censitários $(20 \%)$ entre os 246 existentes na zona urbana do município do Rio Grande ${ }^{21}$, sendo que em cada setor deveriam ser visitados 28 domicílios (1400 residências/50 setores censitários). A seguir, em cada um desses setores, foi realizada uma amostragem aleatória simples, sorteando primeiro uma quadra do setor e depois uma das suas esquinas. Posteriormente, e a partir da esquina sorteada, foi realizada uma amostragem sistemática, iniciando pela primeira residência à esquerda e visitando uma de cada duas residências até atingir as 28 casas previstas por setor. Todas as mulheres com idade igual ou superior a 18 anos residentes nos domicílios selecionados eram entrevistadas. Se alguma das mulheres residentes na casa não se encontrava no momento da entrevista era agendada uma nova visita no horário e dia em que estivesse disponível.

\section{Definição do desfecho e variáveis independentes}

O conhecimento sobre fatores de risco para DCV foi avaliado mediante a pergunta Você (a Sra.) poderia me dizer o que pode levar à angina ou ao infarto? Uma lista de fatores modificáveis e considerados importantes para a DCV, segundo a literatura, foi preparada com antecedência. Estes fatores, extraídos da literatura consultada, incluíam a presença de obesidade, o tabagismo, a pressão alta, o diabetes, o sedentarismo, o estres$\mathrm{se}^{6,7}$ e a ingesta de alimentos com gordura ${ }^{5}$. Após a realização da pergunta, a entrevistada devia responder espontaneamente, e não de forma estimulada com a leitura das opções possíveis, qual os quais os fatores ela acreditava que fossem causadores da DCV. A resposta ou as respostas ditas pelas entrevistadas eram anotadas pelo entrevistador seguindo a lista previamente confeccionada. Aquelas que não se classificavam nas opções previstas eram anotadas separadamente para posterior codificação e análise.

O desfecho, conhecimento sobre fatores de risco para DCV, foi construído a partir da soma do número de fatores de risco para DCV mencionados por cada entrevistada, sendo o mínimo 0 e o máximo 7. Para fins de análise, o escore foi dicotomizado, utilizando como ponto de corte $2 / 3$, o que correspondeu ao percentil 75 da distribuição de respostas obtidas na amostra estudada. Este percentil foi adotado embasado em outras pesquisas já realizadas ${ }^{10,11,22}$.

Como variáveis independentes foram estudadas a idade (em anos completos e posteriormente categorizada), a situação conjugal (classificada em com ou sem cônjuge), a cor da pele (observada pela entrevistadora e classificada posteriormente como branca e negra e outras), a escolaridade (em anos completos e posteriormente categorizada em ensino médio incompleto ou completo), o quartil de renda per capita familiar (calculado a partir da renda familiar dividida pelo número de moradores na casa), sendo o $1^{\circ}$ quartil referente ao grupo menos favorecido economicamente). Entre os hábitos de vida, foram analisados o tabagismo (classificado como não fuma e fuma ou fumava até seis meses antes da entrevista), o uso regular de bebidas alcoólicas (definido como a ingestão de qualquer bebida alcóolica uma vez ao mês ou menos) e o sedentarismo (avaliada através de pergunta que inqueria se a entrevistada participava de qualquer atividade ou programa de educação física). Perguntou-se também sobre a existência de DCV, 
DM (tipo I e tipo II) e HAS autorreferidas e diagnosticadas pelo médico. A coleta dessas variáveis de morbidade permitiu analisar o efeito que o fato de ser portador desse tipo de doença tem sobre o conhecimento dos fatores de risco para DCV, e permitindo o ajuste com outras variáveis. Se bem que era esperado que portadores dessas doenças tivessem um melhor conhecimento dos fatores envolvidos, essa associação não é necessariamente redundante, uma vez que muitos doentes podem desconhecer os fatores de risco que levam à doença, seja por falta de informação ou por desinteresse. A não inclusão desses fatores no modelo de análise limitaria a compreensão dos fatores que estão associados a um melhor conhecimento dos fatores de risco.

\section{Coleta e análise dos dados}

A coleta dos dados ocorreu por meio de um questionário pré-codificado e testado e que continha todas as variáveis citadas anteriormente. O estudo piloto foi realizado em um dos setores censitários não sorteados. As entrevistas foram realizadas por seis entrevistadoras treinadas e com padronização na aplicação dos questionários e na logística do estudo. O questionário era aplicado, conforme explicado antes, a todas as mulheres com 18 anos ou mais residentes no domićlio. Havendo recusa para realizar a entrevista ou não se encontrando a pessoa no domicílio eram realizadas outras duas tentativas antes de se considerar como uma perda. As perdas não foram repostas. Foram refeitas $10 \%$ de todas as entrevistas para avaliar a qualidade da informação obtida e a confiabilidade do instrumento aplicado. As entrevistas foram realizadas após a assinatura do Termo de Consentimento Livre e Esclarecido $^{23}$. O projeto de pesquisa foi aprovado pela Comissão de Ética e Pesquisa da Universidade Federal do Rio Grande.

Os dados obtidos foram duplamente digitados no programa EpiData 3.1 (EpiData Association, Dinamarca) e para as análises estatísticas foi utilizado o pacote estatístico Stata, versão 11 (StataCorp., CollegeStation, Estados Unidos). A análise de dados incluiu a descrição da amostra e a prevalência dos fatores de risco de DCV conhecidos. Posteriormente, foi criado um escore com a soma dos fatores de risco mencionados. Esse escore foi transformado em uma variável dicotômica, utilizando para isso o ponto de corte obtido pelo percentil 75 da distribuição de escores, e que correspondeu a um valor igual ou superior a três. Após, foi realizada a análise bivariada para estudar as associações entre as variáveis de interesse e o desfecho dicotomizado, sendo calculadas as Razões de Prevalências brutas (RP) e seus respectivos intervalos de confiança de 95\% (IC95). Posteriormente, na análise multivariável, foi utilizada a Regressão de Poisson de tipo "backward stepwise", e com variância robusta utilizando o comando "cluster" do Stata. Calcularam-se as RP ajustadas e seus IC95\%, seguindo um modelo hierárquico de análise ${ }^{24}$ que compreendeu três níveis de determinação. No nível mais distal encontravam-se as variáveis sociodemográficas: idade, situação conjugal, cor da pele, renda e escolaridade. No segundo nível foram incluídos os hábitos de vida, como tabagismo, uso regular de bebidas de álcool e o sedentarismo. No terceiro nível, incluíram-se a presença das doenças crônicas HAS, DM e DCV. Todas as variáveis de cada nível eram ingressadas em bloco no modelo. Aquelas com um p menor ou igual a 0,20 eram mantidas para o ajuste com as variáveis do nível seguinte. Esse procedimento era realizado repetidamente até o último nível hierárquico. Foi avaliada a possibilidade de colinearidade, o que não ocorreu em nenhum dos modelos construídos. O teste estatístico utilizado foi o de Wald. No caso de variáveis politômicas ordinais e cujas RP apresentavam uma relação de aumento ou redução progressiva dos coeficientes foi utilizado o teste de Wald para tendência linear. Se as RP não apresentavam ordenamento algum era aplicado o teste de Wald para heterogeneidade. Em todos os testes de significância foi considerado um valor de p menor a 0,05 de um teste bicaudal.

\section{Resultados}

Foram identificadas 1.626 mulheres, das quais 33 não foram encontradas ou recusaram-se a responder, totalizando $2 \%$ de perdas. Assim a amostra ficou constituída por 1.593 mulheres. A idade média do grupo foi de 44,6 anos (DP $=17,8)$, com predomínio de participantes de cor da pele branca $(70,8 \%)$ e que viviam sem o cônjuge $(54,4 \%)$. Quanto aos fatores socioeconômicos, a média de renda variou de $\mathrm{R} \$ 213,5$ a 2421,4 e a maioria possuía o ensino médio incompleto $(61,0 \%)$.

O tabagismo foi relatado por mais de um quarto das mulheres e o consumo de álcool ocorreu em uma em cada 10 entrevistadas. O sedentarismo foi identificado em $37,4 \%$ da amostra e a HAS foi a morbidade referida mais frequente $(30,6 \%)$, enquanto a DM e a DCV situaram-se em $5,5 \%$ e $4,3 \%$ respectivamente (Tabela 1 ). 
Tabela 1. Descrição da amostra de mulheres conforme as características estudadas. Rio Grande, Rio Grande do Sul, Brasil, 2011 ( $\mathrm{n}=1593)$.

\begin{tabular}{|c|c|c|}
\hline Características & $\mathbf{n}$ & $\%$ \\
\hline \multicolumn{3}{|l|}{ Idade (anos) } \\
\hline $18-24$ & 262 & 16,5 \\
\hline $25-44$ & 547 & 34,3 \\
\hline $45-64$ & 548 & 34.4 \\
\hline 65 ou mais & 236 & 14,8 \\
\hline \multicolumn{3}{|l|}{ Situação conjugal } \\
\hline Com cônjuge/companheiro & 726 & 45,6 \\
\hline Sem cônjuge/companheiro & 867 & 54,4 \\
\hline \multicolumn{3}{|l|}{ Cor da pele } \\
\hline Branca & 1128 & 70,8 \\
\hline Negra e outras & 465 & 29,2 \\
\hline Renda/quartil ( $\mathrm{n}=1580)$ & \multicolumn{2}{|r|}{ Média (DP) } \\
\hline $1^{\circ}$ quartil & 400 & $213,5(71,5)$ \\
\hline $2^{\circ}$ quartil & 391 & $440,8(67,8)$ \\
\hline $3^{\circ}$ quartil & 423 & $745,3(134,9)$ \\
\hline $4^{\text {o }}$ quartil & 366 & $2421,4(1506,9)$ \\
\hline \multicolumn{3}{|l|}{ Escolaridade } \\
\hline Ensino médio incompleto & 972 & 61,0 \\
\hline Ensino médio completo & 621 & 39,0 \\
\hline \multicolumn{3}{|l|}{ Tabagismo } \\
\hline Não & 1158 & 72,7 \\
\hline Sim & 435 & 27,3 \\
\hline \multicolumn{3}{|c|}{ Uso regular de bebidas alcoólicas } \\
\hline Não & 1405 & 88,2 \\
\hline Sim & 188 & 11,8 \\
\hline \multicolumn{3}{|l|}{ Sedentarismo } \\
\hline Não & 998 & 62,6 \\
\hline Sim & 595 & 37,4 \\
\hline \multicolumn{3}{|l|}{ Doença Cardiovascular } \\
\hline Não & 1524 & 95,7 \\
\hline Sim & 68 & 4,3 \\
\hline \multicolumn{3}{|l|}{ Diabetes Mellitus } \\
\hline Não & 1504 & 94,5 \\
\hline Sim & 88 & 5,5 \\
\hline \multicolumn{3}{|l|}{ Hipertensão Arterial Sistêmica } \\
\hline Não & 1105 & 69,4 \\
\hline Sim & 487 & 30,6 \\
\hline
\end{tabular}

$\mathrm{DP}=$ Desvio-Padrão.

Cerca da metade das entrevistadas identificou a pressão alta e o fumo como fatores de risco para DCV (50,8\% e $47,8 \%$ respectivamente). O reconhecimento dos outros fatores de risco pesquisados foi baixo, sendo: $33,6 \%$ para a ingestão de alimentos com gordura, $29,3 \%$, para o estresse, $20,8 \%$, para o sedentarismo, $14,9 \%$, para a obesidade e $6,4 \%$ para a DM. Onze por cento de todas as entrevistadas não conhecia nenhum dos sete fatores de risco associados com a DCV.
A prevalência do desfecho, definido como conhecer três ou mais fatores de risco, foi 33\% (IC95 31,0\% - 35,6). Na análise bruta, todas as variáveis estudadas, com exceção de cor da pele, uso de álcool e ser portadora de alguma das doenças crônicas, associaram-se ao desfecho. Após ajuste mediante a análise multivariada, algumas modificações importantes ocorreram. No primeiro nível, permaneceu associada ao desfecho a idade, sendo que as mulheres na faixa etária de 25 a 44 anos tiveram uma probabilidade $36 \%$ maior e as de 45 a $6412 \%$ maior, comparadas com o grupo mais jovem. Por ter um p menor a 0,20 , a variável situação conjugal foi mantida para ajuste com o nível seguinte. A cor da pele, que na bruta não se associou significativamente, após ajuste passou a sê-lo, sendo que as mulheres não brancas apresentaram um melhor conhecimento (RP $=1,18$; IC95 1,01 - 1,37), do que as de cor de pele branca e outras. No mesmo nível, e ajustado com os outros fatores, a escolaridade e a renda permaneceram associados a um maior conhecimento. As mulheres com ensino médio completo, comparadas com as menos escolarizadas, apresentaram uma probabilidade $28 \%$ maior $(\mathrm{RP}=1,28$; IC95 1,10 - 1,49). No caso da renda evidenciouse uma tendência linear entre os quartis, sendo que o conhecimento aumentou na medida em que ascendeu o quartil, chegando a ser três vezes maior no $4^{\circ}$ em relação ao primeiro $(\mathrm{RP}=2,97$; IC95 2,04 - 4,30). No segundo nível, permaneceu associado significativamente o sedentarismo, com uma redução do conhecimento de 17\%, em comparação com as não sedentárias $(\mathrm{RP}=0,83$; IC95 0,70 - 0,99). Finalmente, no último nível, a condição de ser portadora de DCV, e que na bruta não teve associação significativa, ao ajustar com os outros fatores tornou-se significativa, aumentando a probabilidade de maior conhecimento dos fatores de risco em 50\% $(\mathrm{RP}=1,50$; IC95 1,02-2,20). As outras morbidades referidas, DM e HAS, continuaram sem apresentar efeito significativo sobre o desfecho (Tabela 2). Para avaliar se o fator portador de DCV poderia estar modificando as RP dos fatores pertencentes aos níveis anteriores, foi rodado o mesmo modelo com as variáveis do modelo final, e as RP obtidas foram comparadas com aquelas antes e depois do ajuste com o fator portador de DCV. Os resultados (não detalhados) mostraram que não houvera mudanças significativas nas magnitudes dos fatores dos níveis anteriores entre os dois modelos, indicando que a possiblidade de viés de colisão é bastante improvável. 
Tabela 2. Análise bruta e ajustada dos efeitos das variáveis independentes sobre a prevalência de conhecimento de três ou mais fatores de risco modificáveis para doença cardiovascular. Rio Grande, Rio Grande do Sul, Brasil, $2011(n=1593)$.

\begin{tabular}{|c|c|c|c|c|c|}
\hline Nível/Variável & $\begin{array}{c}\text { Prevalência de } \\
\text { conhecimento } 3 \\
\text { ou mais fatores } \\
\text { Percentil } 75(\%)\end{array}$ & $\begin{array}{c}\text { Bruta } \\
\text { RP (IC95\%) }\end{array}$ & $\begin{array}{c}\text { Valor } \\
\text { p }\end{array}$ & $\begin{array}{c}\text { Ajustada } \\
\text { RP (IC95\%) }\end{array}$ & $\begin{array}{c}\text { Valor } \\
\text { p }\end{array}$ \\
\hline \multicolumn{6}{|l|}{ I nível } \\
\hline Idade $(\operatorname{anos})^{c}$ & & & $0,004^{\mathrm{a}}$ & & $0,001^{\circ}$ \\
\hline $18-24$ & $76(29,0)$ & 1,00 & & 1,00 & \\
\hline $25-44$ & $212(38,8)$ & $1,34(1,06-1.69)$ & & $1,36(1,09-1,69)$ & \\
\hline $45-64$ & $173(31,6)$ & $1,09(0,86-1,38)$ & & $1,12(0,88-1,42)$ & \\
\hline 65 ou mais & $70(29,7)$ & $1,02(0,75-1,39)$ & & $0,97(0,72-1,32)$ & \\
\hline Situação conjugal & & & & & 0,08 \\
\hline Com cônjuge & $219(30,2)$ & 1,00 & 0,02 & 1,00 & \\
\hline Sem cônjuge & $312(36,0)$ & $1,19(1,04-1.37)$ & & $1,16(0,98-1,37)$ & \\
\hline Cor da pele & & & & & 0,03 \\
\hline Branca & $370(32,8)$ & 1,00 & 0,5 & 1,00 & \\
\hline Negra e outras & $161(34,6)$ & $1,06(0,91-1,23)$ & & $1,18(1,01-1,37)$ & \\
\hline $\operatorname{Renda}^{c}$ & & & & & $<0,001^{\mathrm{b}}$ \\
\hline $1^{\circ}$ quartil & $67(16,7)$ & 1,00 & $<0,001^{\mathrm{b}}$ & 1,00 & \\
\hline $2^{\circ}$ quartil & $101(25,8)$ & $1,54(1,17-2,03)$ & & $1,54(1,16-2,06)$ & \\
\hline $3^{\circ}$ quartil & $163(38,5)$ & $2,30(1,79-2,95)$ & & $2,15(1,63-2,83)$ & \\
\hline $4^{\circ}$ quartil & $193(52,7)$ & $3,15(2,48-4,00)$ & & $2,97(2,04-4,30)$ & \\
\hline Escolaridade $^{c}$ & & & & & 0,003 \\
\hline Ensino médio incompleto & $255(26,2)$ & 1,00 & $<0,001$ & 1,00 & \\
\hline Ensino médio completo & $276(44,4)$ & $1,69(1,48-1,94)$ & & $1,28(1,09-1,51)$ & \\
\hline \multicolumn{6}{|l|}{ II nível } \\
\hline Tabagismo & & & $<0,001$ & & 0,3 \\
\hline Não & $421(36,4)$ & 1,00 & & 1,00 & \\
\hline Sim & $110(25,3)$ & $0,69(0,58-0,83)$ & & $0,92(0,78-1,09)$ & \\
\hline Uso de bebidas alcoólicas & & & 0,55 & & 0,3 \\
\hline Não & $472(33,6)$ & 1,00 & & 1,00 & \\
\hline Ao menos 1 vez ao mês & $59(31,4)$ & $0,93(0,74-1,16)$ & & $0,85(0,65-1,11)$ & \\
\hline Sedentarismoc & & & $<0,001$ & & 0,04 \\
\hline Não & $374(37,5)$ & 1,00 & & 1,00 & \\
\hline Sim & $157(26,4)$ & $0,70(0,60-0,82)$ & & $0,83(0,70-0,99)$ & \\
\hline \multicolumn{6}{|l|}{ III nível } \\
\hline Doença cardiovascular ${ }^{c}$ & & & 0,53 & & 0,04 \\
\hline Não & $506(33,2)$ & 1,00 & & 1,00 & \\
\hline Sim & $25(36,8)$ & $1,10(0,80-1,52)$ & & $1,50(1,02-2,20)$ & \\
\hline Diabetes Mellitus & & & 0,75 & & 0,7 \\
\hline Não & $503(33,4)$ & 1,00 & & 1,00 & \\
\hline $\operatorname{Sim}$ & $28(31,8)$ & $0,95(0,69-1,30)$ & & $1,06(0,83-1,36)$ & \\
\hline Hipertensão Arterial & & & 0,33 & & 0,3 \\
\hline Não & $377(34,1)$ & 1,00 & & 1,00 & \\
\hline $\operatorname{Sim}$ & $154(31,6)$ & $0,93(0,79-1,08)$ & & $1,09(0,91-1,31)$ & \\
\hline
\end{tabular}

${ }^{\text {a }}$ Teste de Wald de heterogeneidade; ${ }^{\mathrm{b}}$ Teste de Wald de tendência linear; ${ }^{\mathrm{c}}$ modelo final.

\section{Discussão}

Estudos de base populacional no Brasil que estudem o conhecimento que tem a população geral a respeito dos principais fatores de risco modifi- cáveis para DCV não são tão frequentes, limitando-se a maior parte a identificar apenas alguns deles. O presente estudo, realizado em município no extremo sul do Brasil, com a finalidade de avaliar o conhecimento entre mulheres dos prin- 
cipais fatores de risco associados à DCV, revelou que a proporção que conhecia três ou mais fatores foi de apenas 33,0\% e que a distribuição do conhecimento de três ou mais fatores foi maior entre aquelas mais escolarizadas e cresceu linearmente na medida em que aumentava o quartil de renda familiar per capita, demonstrando assim a presença de iniquidade no conhecimento dos fatores de risco para DCV.

Devem ser consideradas algumas potenciais limitações no presente estudo. Em primeiro lugar, o tipo de delineamento não permite estabelecer relações causais e os fatores identificados devem ser vistos apenas como associações. É claro que para determinados fatores o delineamento não deve ter sido uma limitação. É o caso dos socioeconômicos, como renda ou escolaridade, uma vez que temporalmente parece pouco provável que o conhecimento sobre o desfecho neste caso possa determinar a posteriori ambos. Outra limitação é que por ser um estudo de base populacional restrito às mulheres, seus resultados não podem ser extrapolados para os homens. Em terceiro lugar, a respeito da cor da pele, não foi utilizada a autodeclaração do entrevistado e sim a identificação pelo entrevistador. Por esse motivo pode ter havido um viés de informação. Existe uma tendência do entrevistador adjudicar mais a cor branca do que quando a cor é autodeclarada. Em quarto lugar, no caso das morbidades, em particular DCV, e fatores de risco autorreferidos, como fumo ou sedentarismo, pode ser arguido que existisse um viés de colisão, entre as doenças, os fatores e o conhecimento destes ${ }^{25}$. Em princípio, devemos esclarecer que a forma em que se selecionaram os participantes (estudo transversal com amostra aleatória) deve ter diminuído a possibilidade do viés de colisão. Mas, como esse viés pode também surgir ao realizar as análises durante as estratificações, não podemos descartar sua existência. Contudo, devemos considerar que as morbidades entraram no último nível de hierárquico de análise, e que as RP dos fatores dos níveis anteriores não se modificaram substancialmente após o ajuste com esse fator. Portanto, se o viés de colisão existiu, ele não deve ter afetado de forma considerável os resultados observados nas outras variáveis. Finalmente, outro aspecto metodológico que deve ser discutido é a validade das informações obtidas sobre morbidades referidas, como a presença de hipertensão arterial. Sobre este ponto existem suficientes evidências de que esse tipo de informação referida pelo paciente apresenta validade, reduzindo assim a possibilidade de perda da validez ${ }^{26}$.
Existem aspectos do estudo que fortalecem os resultados obtidos. Entre eles citamos a baixa frequência de perdas (2\%), o que acrescenta validade interna ao estudo. Outro aspecto que fortalece o estudo é a forma de avaliar o conhecimento sobre os fatores de risco. Quando se deseja estimar o conhecimento para que ocorra uma mudança é fundamental avaliar o que as pessoas sabem por elas mesmas, sem fazer qualquer tipo de indução sobre possíveis respostas. Assim, neste estudo, se optou pela resposta espontânea a partir da realização da pergunta, e não induzida através da leitura, ou apresentação gráfica dos fatores possíveis, o que com certeza aumentaria o nível de conhecimento observado. Desta forma, a informação obtida é aquela que realmente o indivíduo possui quando decidiria iniciar o processo de mudança.

A prevalência encontrada de $33,0 \%$ está abaixo do que foi evidenciado por outros estudos que utilizaram o mesmo critério para definir o desfecho (percentil 75). Khan et al. ${ }^{11}$ e Saeed et al. ${ }^{27}$ encontraram prevalências de conhecimento de $42,0 \%$ e $41,0 \%$, respectivamente. A maior prevalência encontrada pode ser explicada pelo tipo de amostra utilizada, uma vez que os dois estudos foram realizados com pacientes de serviços de saúde, e as opções foram lidas e não respondidas espontaneamente. Essa explicação é viável, porque quando realizamos uma subanálise do nosso estudo utilizando unicamente as portadoras de DCV, a prevalência subiu para $37,0 \%$, valor bem mais próximo ao encontrado pelos dois outros estudos. Além dessa diferença na população alvo, deve-se considerar a geográfica e cultural, uma vez que esses estudos foram realizados em outros países.

O estudo evidenciou que a pressão alta e o fumo foram os fatores de risco mais frequentemente identificados (uma de cada duas mulheres avaliadas), sendo este resultado consistente com o encontrado por outras pesquisas dentro ${ }^{28} \mathrm{e}$ fora do Brasil ${ }^{11,27,29-31}$, mas com prevalências inferiores às que estes relataram. Novamente, devemos lembrar que no nosso estudo a identificação dos fatores foi espontânea e não estimulada, o que certamente reduz a proporção de indivíduos que podem responder corretamente.

O maior conhecimento desses fatores pode, de certa forma, ser explicado pela ênfase proporcionada no Brasil, através dos meios de comunicação, profissionais e serviços de saúde às campanhas populacionais de esclarecimento sobre os malefícios do cigarro, bem como da necessidade de verificação da pressão alta. 
Merece atenção a ausência de conhecimento de uma grande parte das mulheres de diversos fatores importantes na patogênese da DCV. O papel dos fatores de risco diabetes e obesidade na DCV foram desconhecidos para $94,0 \%$ e $85,0 \%$, respectivamente, das mulheres. Ainda mais, $11 \%$ das entrevistadas não foram capazes de identificar nenhum fator. Esses achados são consistentes com outros estudos ${ }^{11,27,31-33}$, ainda que se considerem as diferenças culturais entre países. Essa falta de conhecimento sobre o papel da diabetes como fator de risco para DCV pode ocorrer também pela não percepção por parte da população que uma doença, como a diabetes, pode ser também um fator de risco para outra. Essa representação decorre da interpretação que as pessoas fazem a partir das crenças associadas aos grupos sociais, e envolve tanto processos coletivos como vivências individuais a partir das experiências de doença que experimentam, e assim pode mudar geográfica e socialmente ${ }^{34}$.

Com respeito à idade, este estudo evidenciou que mulheres na faixa etária de 25 a 44 e de 45 a 64, ou seja, adultos jovens e maduros, apresentaram uma maior probabilidade de conhecer os fatores de risco, quando comparadas às mais jovens, e os idosos mostraram uma redução de $3 \%$. Estas associações e a direção delas foram observadas também por outros estudos ${ }^{28,29}$, e mostraram que os indivíduos adultos maduros, talvez por estarem em uma faixa etária onde as manifestações da DCV se iniciam, teriam um melhor conhecimento dos fatores responsáveis. Já aqueles mais novos, devido a terem uma menor incidência e prevalência dessa doença, apresentariam uma menor preocupação com a ocorrência dessas enfermidades e, portanto, não teriam tanta informação a respeito. Já aqueles indivíduos mais idosos, talvez por terem menor contato e compreensão das mensagens veiculadas, seriam menos propensos a mencionar os fatores de risco, se bem que o efeito observado foi muito pequeno. Já ao fazer uma subanálise com aquelas mulheres portadoras de DCV, a informação sobre os fatores de risco aumentou na medida em que se incrementou os grupos de idade, mostrando a relação entre o crescimento da prevalência da doença na medida em que se envelhece e o aumento do conhecimento dos fatores associados.

O conhecimento dos fatores de risco para DCV é influenciado pelas características sociodemográficas das populações, sendo que aqueles mais carentes o têm menor, evidenciando uma importante iniquidade em saúde ${ }^{33}$. No presente estudo foi encontrada também essa iniquidade.
Encontrou-se uma tendência linear entre a renda e o conhecimento de fatores de risco, sendo que as mulheres do último quartil apresentaram três vezes maior probabilidade de ter mais informação sobre os fatores envolvidos na DCV quando comparadas com aquelas mais pobres do $1^{\circ}$ quartil. Este achado é consistente com outros estudos no Brasil, que avaliaram alguns fatores para DCNT ${ }^{14,28}$, e em outros países ${ }^{10,31,35}$. O resultado pode ser explicado pelo efeito que a renda tem sobre o acesso a informações de qualidade nos meios de comunicação, assim como a melhores cuidados de saúde, incluindo a promoção e a prevenção de doenças.

Da mesma forma, neste estudo foi observado que a escolaridade esteve associada com um maior conhecimento dos fatores de risco, sendo que as mulheres com ensino médio completo ou maior nível de educação tiveram maior probabilidade de conhecer mais fatores de risco. Este achado é evidenciado em pesquisas realizadas no Brasil ${ }^{14,28}$ assim como em outros países ${ }^{10,11,22,29,31,34,36}$. Contudo no presente estudo o efeito observado não foi tão grande (28\%) se comparado com o da renda (de 1,5 a 3x dependendo do quartil). Isso pode indicar que a grande barreira para obter esse conhecimento não é tanto o grau de educação e sim a possibilidade de ter acesso financeiro à informação de boa qualidade, oportuna e pertinente.

A atividade física regular é considerada como um importante fator para prevenção de doenças cardíacas ${ }^{7}$. Este estudo evidenciou que cerca de $80,0 \%$ das mulheres não identificaram a relação entre exercício físico e DCV. Outros estudos realizados fora do Brasil, e que abordaram o conhecimento sobre o papel desse fator na prevenção de doenças, encontraram maiores prevalências ${ }^{11,27,31}$, mas devemos considerar que essa diversidade pode ter surgido por diferenças metodológicas, uma vez que, como explicado anteriormente, na presente pesquisa os fatores eram identificados de forma espontânea, sem a leitura das opções possíveis.

Concluindo, os resultados do nosso estudo evidenciaram que o conhecimento dos sete fatores de risco modificáveis para DCV é baixo entre as mulheres com 18 anos ou mais e que existe iniquidade na distribuição segundo a renda e a escolaridade.

Estes achados apontam para a necessidade de intensificar os programas de educação para prevenção de doenças crônicas e, em particular, para a difusão dos fatores associados com a DCV entre os grupos mais carentes. A importância de 
tal prática se sustenta na evidência de que para que uma pessoa mude seu comportamento, deve existir o conhecimento prévio dos fatores envolvidos responsáveis pela doença, seguido pela consciência de que, sendo portador de tal risco, a persistência nessa conduta pode provocar um dano à saúde. Como foi evidenciado de que existem iniquidades importantes, é imprescindível que, junto aos programas para a população em geral, sejam elaborados outros específicos dirigidos para as mulheres com menores condições socioeconômicas. As unidades básicas de saúde, e em particular a Estratégia da Saúde da Família, podem ter um papel fundamental nesta difusão do conhecimento. Ocorrendo a melhora no grau de discernimento na população geral sobre os danos produzidos por determinados comportamentos e situações de doença, pode-se buscar comportamentos preventivos adequados, que, consequentemente, levem a uma redução da carga de morbidade e mortalidade por DCV em todas as camadas sociais da população.

\section{Colaboradores}

GM Bonotto participou da concepção do estudo, revisão crítica da literatura, análise, interpretação de dados e redação do manuscrito. RAM Sassi esteve envolvido na concepção do estudo, supervisão da coleta de dados, análise, interpretação de dados e revisão. LRO Susin colaborou também na concepção do estudo e revisão do manuscrito.

\section{Referências}

1. Schmidt MI, Duncan BB, Silva GA, Menezes AM, Monteiro CA, Barreto SM, Chor D, Menezes PR. Doenças crônicas não transmissíveis no Brasil: carga e desafios atuais. Lancet [internet] 2011. [acessado 2012 ago 14]. Disponível em: http://download.thelancet.com/flatcontentassets/pdfs/brazil/brazilpor4.pdf

2. Schramm JMA, Oliveira AF, Leite IC, Valente JG, Gadelha AMJ, Portela MC, Campos MR. Transição epidemiológica e o estudo de carga de doença no Brasil. Cien Saude Colet 2004; 9(4):897-908.

3. Moraes SA, Freitas ICM. Doença isquêmica do coração e fatores associados em adultos de Ribeirão Preto, SP. Rev Saude Publica 2012; 46(4):591-601.

4. Mansur AP, Favarato D. Mortalidade por Doenças Cardiovasculares no Brasil e na Região Metropolitana de São Paulo: Atualização 2011. Arq Bras Cardiol 2012; 99(2):755-761

5. Mann JI. Diet and risk of coronary heart disease and type 2 diabetes. Lancet 2002; 360(9335):783-89.

6. Yusuf S, Hawken S, Ôunpuu S, Dans T, Avezum A, Lanas F, McQueen M, Budaj A, Pais P, Varigos J, Lisheng L; INTERHEART Study Investigators. Effect of potentially modifiable risk factors associated with AMI in 52 countries (the INTERHEART study): case-control study. Lancet 2004; 364(9438):937-952.

7. Lanas F, Avezum A, Bautista LE, Diaz R, Luna M, Islam S, Yusuf S; INTERHEART Investigators in Latin America. Risk factors for Acute Myocardial Infarction in Latin America: the INTERHEART Latin American Study. Circulation 2007; 115(9):1067-1074.

8. Dijkstra A, Vries H, Bakker M. Pros and cons of quitting, self-efficacy, and the stages of change in smoking cessation. J Consult Clin Psychol 1996; 64(4):758-763.

9. Prochaska JO, Velicer WF, Rossi JS, Goldstein MG, Marcus BH, Rakowski W, Fiore C, Harlow LL, Redding CA, Rosenbloom D, Rossi SR. Stages of change and decisional balance for 12 problem behaviors. Health Psychol 1994; 13(1):39-46. 
10. Jafary FH, Aslam F, Mahmud H, Waheed A, Shakir M, Afzal A, Qayyum MA, Akram J, Khan IS, Haque IU. Cardiovascular health knowledge and behavior in patient attendants at four tertiary care hospitals in Pakistan - a cause for concern. BMC Public Health 2005; $5: 124$.

11. Khan MS, Jafary FH, Jafar TH, Faruqui AM, Rasool SI, Hatcher J, Chaturvedi N. Knowledge of modifiable risk factors of heart disease among patients with acute myocardial infarction in Karachi, Pakistan: a cross sectional study. BMC Cardiovasc Disord 2006; 6:18.

12. Lynch EB, Liu K, Kiefe CI, Greenland P. Cardiovascular disease risk factor knowledge in young adults and 10-year change in risk factors: the Coronary $\mathrm{Ar}$ tery Risk Development in Young Adults (CARDIA) Study. Am J Epidemiol 2006; 164(12):1171-1179.

13. Sanderson SC, Waller J, Jarvis MJ, Humphries SE, Wardle J. Awareness of lifestyle risk factors for cancer and heart disease among adults in the UK. Patient Educ Couns 2009; 74(2):221-227.

14. Knuth AG, Bielemann RM, Silva SG, Borges TT, Del Duca GF, Kremer MM, Hallal PC, Rombaldi AJ, Azevedo MR. Conhecimento de adultos sobre o papel da atividade física na prevenção e tratamento de diabetes e hipertensão: estudo de base populacional no Sul do Brasil. Cad Saude Publica 2009; 25(3):513-520.

15. Domingues MR, Araújo CLP, Gigante DP. Conhecimento e percepção sobre exercício físico em uma população adulta urbana do Sul do Brasil. Cad Saude Publica 2004; 20(1):204-215.

16. Muniz LC, Schneider BC, Silva ICM, Matijasevich A, Santos IS. Accumulated behavioral risk factors for cardiovascular diseases in Southern Brazil. Rev Saude Publica 2012; 46(3):534-534.

17. Schmidt MI, Duncan BB, Hoffmann JF, Moura L, Malta DC, Carvalho RMSV. Prevalência de diabetes e hipertensão no Brasil baseada em inquérito de morbidade auto-referida, Brasil, 2006. Rev Saude Publica 2009; 43(Supl. 2):74-82.

18. Gigante DP, França GVA, Sardinha LMV, Iser BPM Meléndez GV. Variação temporal na prevalência do excesso de peso e obesidade em adultos: Brasil, 2006 a 2009. Rev Bras Epidemiol 2011; 14(Supl. 1):157-165.

19. Silva GA, Valente JG, Malta DC. Tendências do tabagismo na população adulta das capitais brasileiras: uma análise dos dados de inquéritos telefônicos de 2006 a 2009. Rev Bras Epidemiol 2011; 14(3):103-114.

20. Brasil. Instituto Brasileiro de Geografia e Estatística. Censo demográfico, 2010. [acessado 2013 ago 20]. Disponível em: http://www.censo2010.ibge.gov.br

21. Brasil. Instituto Brasileiro de Geografia e Estatística. Censo demográfico, 2000.[acessado 2010 jan 9]. Disponível em: http://www.censo.ibge.gov.br

22. Fussman C, Rafferty AP, Reeves MJ, Zackery S, Lyon-Callo S, Anderson B. Racial disparities in knowledge of stroke and heart attack risk factors and warning signs among Michigan adults. Ethn Dis 2009; 19(2):128-134.

23. Brasil. Ministério da Saúde (MS). Conselho Nacional de Saúde. Resolução no 196 de 10 de outubro de 1996. Diretrizes e Normas Regulamentadoras de Pesquisas Envolvendo Seres Humanos. Diário Oficial da União 1996; 16 out.
24. Victora CG, Huttly SR, Fuchs SC, Olinto MT The role of conceptual frameworks in epidemiological analysis: a hierarchical approach. Int J Epidemiol.1997; 26(1):224-7.

25. Greenland S. Quantifying biases in causal models; Classical confoundng vs collider-stratification bias. Epidemiology 2003; 14(3):300-306.

26. Chrestani MAD, Santos IS, Matijasevich A. Hipertensão arterial sistêmica autorreferida: validação diagnóstica em um estudo de base populacional. Cad Saude Publica 2009; 25:2395-2406.

27. Saeed O, Gupta V, Dhawan N, Streja L, Shin JS, Ku M, Bhoi S, Verma S. Knowledge of modifiable risk factors of Coronary Atherosclerotic Heart Disease (CASHD) among a sample in India. BMC Int Health Hum Rights 2009; 9:2.

28. Borges TT, Rombaldi AJ, Knuth AG, Hallal PC. Conhecimento sobre fatores de risco para doenças crônicas: estudo de base populacional. Cad Saude Publica 2009; 25(7):1511-1520.

29. Potvin L, Richard L, Edwards, AC. Knowledge of cardiovascular disease risk factors among the Canadian population: relationships with indicators of socioeconomic status. CMAJ 2000; 162(Supl. 9):S5-11.

30. Querales M, Ruiz N, Rojas S, Espinoza M. Nivel de conocimiento sobre factores de riesgo cardiovascular en una comunidad de Naguanagua, Venezuela. Rev Salud Publica 2011; 13(5):759-771.

31. Hamarneh YN, Crealey GE, Mcelnay JC. Coronary heart disease: health knowledge and behaviour. Int J Clin Pharm 2011; 33(1):111-123.

32. Kandula NR, Tirodkar MA, Lauderdale DS, Khurana NR, Makoul G, Baker DW. Knowledge Gaps and Misconceptions About Coronary Heart Disease Among U.S. South Asians. Am J Prev Med 2010; 38(4):439-442.

33. Wartak SA, Friderici J, Lotfi A, Verma A, Kleppel R, Naglieri-Prescod D, Rothberg MB. Patients' knowledge of risk and protective factors for cardiovascular disease. Am J Cardiol 2011; 107(10):1480-1488.

34. Canesqui AM. Os estudos de antropologia da saúde/ doença no Brasil na década de 1990. Cien Saude Colet 2003; 8(1):109-124.

35. Zaman MJ, Patel A, Jan S, Hillis GS, Raju PK, Neal B, Chow CK. Socio-economic distribution of cardiovascular risk factors and knowledge in rural India. Int $J$ Epidemiol 2012; 41(5):1302-1314.

36. Dodani S, Mistry R, Farooqi M, Khwaja A, Qureshi R, Kazmi K. Prevalence and awareness of risk factors and behaviours of coronary heart disease in an urban population of Karachi, the largest city of Pakistan: a community survey. J Public Health 2004; 26(3):245-249.

Artigo apresentado em 30/11/2014

Aprovado em 04/06/2015

Versão final apresentada em 08/06/2015 